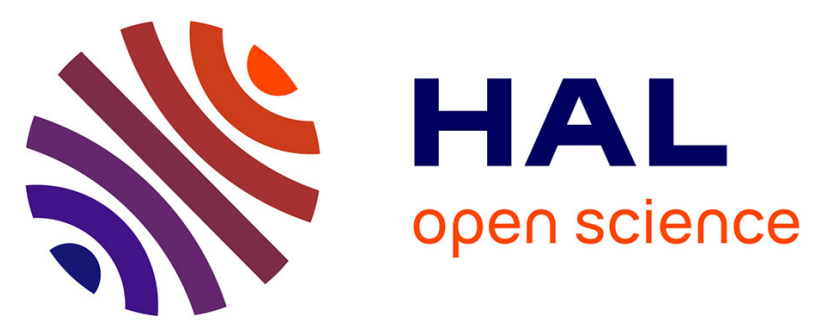

\title{
Mirror, Mirror on My Phone: Investigating Dimensions of Self-Face Perception Induced by Augmented Reality Filters
}

Rebecca Fribourg, Etienne Peillard, Rachel Mcdonnell

\section{- To cite this version:}

Rebecca Fribourg, Etienne Peillard, Rachel Mcdonnell. Mirror, Mirror on My Phone: Investigating Dimensions of Self-Face Perception Induced by Augmented Reality Filters. 2021 IEEE International Symposium on Mixed and Augmented Reality (ISMAR), Oct 2021, Bari, Italy. 10.1109/ISMAR52148.2021.00064 . hal-03378222

\author{
HAL Id: hal-03378222 \\ https://hal.science/hal-03378222
}

Submitted on 18 Oct 2021

HAL is a multi-disciplinary open access archive for the deposit and dissemination of scientific research documents, whether they are published or not. The documents may come from teaching and research institutions in France or abroad, or from public or private research centers.
L'archive ouverte pluridisciplinaire HAL, est destinée au dépôt et à la diffusion de documents scientifiques de niveau recherche, publiés ou non, émanant des établissements d'enseignement et de recherche français ou étrangers, des laboratoires publics ou privés.

\section{(1) (1) $\$$}

Distributed under a Creative Commons Attribution - NonCommercial - NoDerivatives 44.0 


\section{Mirror, Mirror on My Phone: Investigating Dimensions of Self-Face Perception Induced by Augmented Reality Filters}

\author{
Rebecca Fribourg* \\ School of Computer Science and \\ Statistics \\ Trinity College Dublin
}

\author{
Etienne Peillard ${ }^{\dagger}$ \\ IMT Atlantique, Lab-STICC, \\ UMR CNRS 6285 \\ Brest, France
}

\author{
Rachel McDonnell* \\ School of Computer Science and \\ Statistics \\ Trinity College Dublin
}

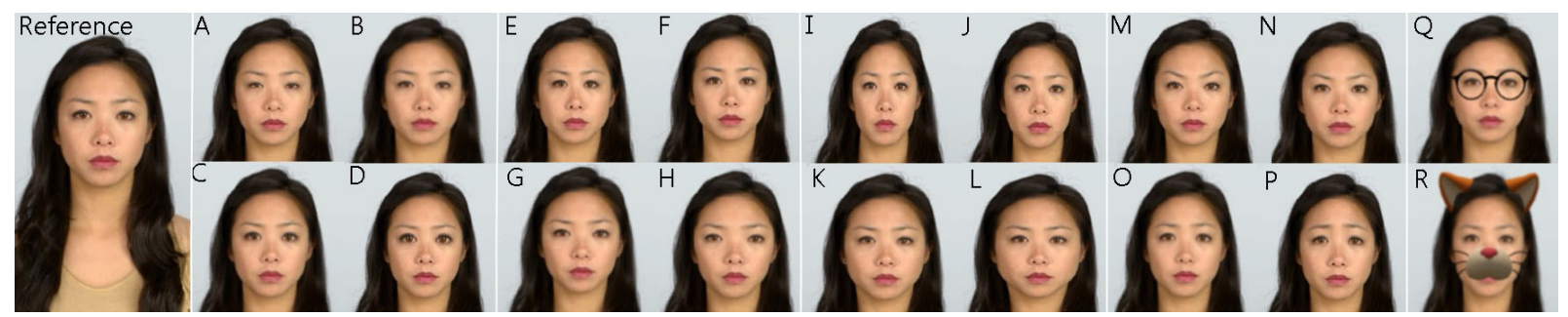

Figure 1: Aggregations of all the filters developed and used in this paper: very small eyes, small eyes, big eyes and very big eyes (A, $B, C, D)$. Very close eyes, close eyes, spaced eyes, very spaced eyes (E, F, G, H). Very narrow face, narrow face, large face, very large face (I, J, K, L). Very inward eyebrows, inward eyebrows, outward eyebrows, very outward eyebrows (M, N, O, P). Realistic AR

\section{ABStRACT} add-on (Q), Unrealistic AR add-on (R).

The main use of Augmented Reality (AR) today for the general public is in applications for smartphones. In particular, social network applications allow the use of many AR filters, modifying users' environments but also their own image. These AR filters are increasingly and frequently being used and can distort in many ways users' facial traits. Yet, we still do not know clearly how users perceive their faces augmented by these filters. In this paper, we present a study that aims to evaluate the impact of different filters, modifying several facial features such as the size or position of the eyes, the shape of the face or the orientation of the eyebrows, or adding virtual content such as virtual glasses. These filters are evaluated via a self-evaluation questionnaire, asking the participants about the personality, emotion, appeal and intelligence traits that their distorted face conveys. Our results show relative effects between the different filters in line with previous results regarding the perception of others. However, they also reveal specific effects on self-perception, showing, inter alia, that facial deformation decreases participants' credence towards their image. The findings of this study covering multiple factors allow us to highlight the impact of face deformation on user perception but also the specificity related to this use in AR, paving the way for new works focusing on the psychological impact of such filters.

Index Terms: Human-centered computing-Human computer interaction (HCI) - Interaction paradigms - Mixed / augmented reality; Human-centered computing-Human computer interaction (HCI) - HCI design and evaluation methods-User studies

\section{INTRODUCTION}

Augmented Reality, as seen through the prism of the general public, mainly concerns today applications that are available on smartphones, and use the stream of the phone camera to superimpose

\footnotetext{
*e-mail: rebecca.fribourg@tcd.ie

†e-mail: etienne.peillard@imt-atlantique.fr

†e-mail: ramcdonn@tcd.ie
}

virtual content. For young people, applications such as SnapChat or Instagram have taken on a central role in personalised augmented reality. Users of these applications are able to engage in real-time face figure distortion (e.g., making the eyes look bigger) and feature addition (e.g., adding dog ears on top of a user's face) which are enabled by a variety of available filters. Filtered faces are today some of the most predominant photos on social media [14], and not only users spend some time looking at themselves through these filters while taking selfies, they can as well use these filters on video calls or video conference with applications such as SnapCamera ${ }^{1}$ or less recent Live! Cam Avatar ${ }^{2}$ application, increasing their exposure to this altered self-image.

In recent years, such filters have received an increased interest in sociological studies, exploring for instance the long term impact of "beauty filters" on self-perception and self-esteem [4, 25]. However, these studies tend to focus on the filters designed to increase users self-perception of attractiveness or cuteness and to study long term effects. Up to this day, a lack of knowledge remains on how users perceive their face when distorted by a large variety of filters and on a short term basis.

Face perception has been at the center of a consequent core of research, from which studies have highlighted that the appeal, intelligence and personality perception of human faces was strongly influenced by specific facial features $[2,16,17]$. In particular, the facial width-to-height ratio (FWHR), eye size and the distance between the eyes was found to influence the perception of,respectively, dominance, trustworthiness and aggressiveness [1,6,29], dominance and trustworthiness $[10,35]$, and intelligence in a human face [12]. These features were also found to influence the perception of attractiveness in human faces, having for instance individuals with larger FWHRs judged less attractive [6]. Furthermore, specific facial features have been shown to allow humans to successfully recognize expressions of emotion, showing for instance, that for fear, surprise, anger and sadness the regions around the eyes have the highest weights [32].

While the depicted studies highlighted valuable insights on the link between facial features and the perception of human faces, they tackle the perception of other peoples faces. Yet, self-face percep-

\footnotetext{
${ }^{1}$ https://snapcamera.snapchat.com/

${ }^{2}$ https://www.bbc.com/news/technology-56010156
} 
tion differs from the perception of other faces as it involves different cognitive processes [26]. Felisberti et al. explored the mechanism of self-face perception with a focus on attractiveness, with one study revealing preferences for eye size enhancement among participants attempting to improve their own attractiveness via digital image alteration [4]. This study however did not explore the link between self-face features and self-face perception of personality traits, as done in face perception studies. As of today, it therefore remains unclear how one perceives appeal, personality and intelligence of one's own face depending on specific facial feature alterations. Because filters that distort face features, as created in Snapchat or Instagram applications, are increasingly being used, we believe that it is important to understand how users perceive their face through those filters. In addition, the use of AR filters in videoconferencing could be used to modify users' perception and potentially also impact their behavior. While self-face is perceived differently than other's faces [26], it is unclear whether facial features are also linked with appeal and personality perception of human faces when ones looks at one's own face. Indeed, when participants in previous studies would evaluate dominance, trustworthiness or other personality features, they would look at pictures of people they do not know, while when we look at ourselves, this perception is influenced by the knowledge we have of ourselves and our individual differences. However, previous works have highlighted the malleability of the self, showing that self-face representation can be altered to include another person's facial features [23]. This encourages us to hypothesize that despite the inner knowledge of one's self, users may be inclined to associate self-facial distortions similarly with perceived personality, appeal and intelligence when looking at themselves through filters as when looking at other faces.

To explore this hypothesis, we conducted an experiment in which participants' facial features were modulated through the use of specifically designed Snapchat filters in order to evaluate their selfface perception of appeal, personality, intelligence and emotion. More precisely, we applied on participants face distortions on facial features that were commonly addressed in face perception studies: the size of eyes, face width, eyes spacing and eyebrows orientation. Each of these filters were applied in two directions (e.g., eyes size could be either big or small), and two levels of intensity, as we were interested if the strength of the filter would impact self-recognition and by extent facial traits judgements of self altered face. Moreover, as filters often involve the addition of virtual content on the face, we added two filters that would add something to the face, one realistic (a pair of glasses) and one non realistic but common in Snapchat or Instagram filters (cat ears and whiskers).

\section{Related Work}

Augmented reality is a powerful technology that allows physical environment to be overlaid with virtual content in real-time. While there are a wide range of AR devices and applications, "augmented mirrors" [9] are extremely popular with the general public, where users view their mirrored image on-screen, and virtual content can be added, or distortions to their facial image can be applied. Among these applications, some are dedicated to marketing purposes (e.g., trying commercial products on oneself, such as virtual sunglasses [34]), while others are more for entertainment or to use in social media (SnapChat or Instagram), and offer a wide range of modifications to apply, such as virtual content superimposition (e.g., cat ears) or distortion (eyes made bigger). Such applications have received some interest in the last years in studies about selfperception through AR mirrors and the relationship with customer behaviour. However, previous work did not investigate in detail how users would perceive their face through these AR mirrors. Studies on face perception have highlighted many insights on how we perceive others faces, and interpret these as personality or emotions. However, these studies did not address the perception of facial traits while observing one's own face and the way users perceive facial traits while looking at themselves in an AR mirror remains unclear. In the first section, we present studies about face perception including self-face and other-face perception and in a second section, we present research about self-perception in augmented reality.

\subsection{Facial Traits Perception of Real Faces}

Human faces are processed in such a way that we instantly interpret personality traits, emotions or evaluate attractiveness and appeal. Through this mechanism, the appearance of each facial feature is known to have an effect on the way facial traits are perceived [2]. More precisely, the traits perceived on faces can be divided into several categories: personality traits, appeal or attractiveness, intelligence and emotions, and are respectively influenced by different facial features. For personality portrayal, the facial width-to-height ratio (FWHR), i.e., bizygomatic width of the face divided by height of the face, was shown to greatly influence the perception of threat, and dominance in faces. People with wider faces were judged more threatening and more dominant, especially for males [6]. Moreover, male faces with higher FWHR tended to be judged less trustworthy [22]. The size of eyes was also found to influence the perception of personality, where faces with larger eyes were perceived as more honest [35] and less dominant [10] than faces with small eyes. Eyes can also be linked with perceived aggressiveness, where smaller eyes are perceived more threatening [11]. The same facial features were also found to affect the perception of attractiveness in human faces. For instance, wider faces tend to be perceived less attractive [6]. As for the size of eyes, studies found that bigger eyes tended to be associated with higher perceived attractiveness [24]. On the other hand, eyes were also associated with perceived intelligence, regarding their inter-distance. Faces that are perceived as highly intelligent had a wider distance between the eyes [12]. In this study, FWHR also seemed to influence perceived intelligence, with longer faces being attributed higher perceived intelligence ratings. Furthermore, specific facial features have been shown to allow humans to successfully recognize expressions of emotion $[2,32]$, showing that eyes and the mouth were of high influence, but also that more precise action units on the face could influence the perception of specific emotions. For instance, for fear, surprise, anger and sadness the regions around the eyes have the highest weights and there is a tendency to focus more on the brows in sadness [32].

Other studies explored how additional items placed on the face, such as glasses, could impact personality, appeal and intelligence perception $[3,15]$. In particular, Leder et al. [15] showed that people may judge faces with glasses as less attractive, but more trustworthy and intelligent.

\subsection{Facial Traits Perception of Virtual Faces}

The perception of facial traits of virtual humans has been addressed in previous work $[5,31]$, exploring the perception of facial feature manipulation for trait portrayal. Interestingly, while research from Neuroscience suggests that real and synthetic faces are processed by the brain in a similar manner [28], the interpretation of virtual faces in terms of personality and appeal judgements tend to vary from real faces. Wang et al. [31] used Second Life characters to explore personality perception, and in contrast to human face studies, they found no link between eye sizes and dominance ratings, and wider faces were not judged to be less trustworthy. More interestingly, they found that wider faces were perceived as less aggressive compared to narrow faces, which is the opposite finding as for human faces. Additionally, they noticed that when a virtual character appeared eerie, trait judgements were either absent or inverted and they argued that it could be explained by reaching a negative Uncanny Valley effect [19]. Ferstl et al. [5] investigated the same question as Wang et al. but with more realistic feature sizes. They found that narrow faces were perceived more aggressive, more dominant and less 
trustworthy, and wider faces were perceived less trustworthy and less attractive. This study supported the finding that virtual faces are perceived differently than human faces.

\subsection{Self-Facial Traits Perception}

Overall, previous research has brought valuable insights on how we perceive human and virtual faces features. Yet, the way we perceive other faces and our own face is not the same and involves different cognitive processes [4]. Felisberti et al. explored the mechanism of self-face perception with a focus on attractiveness, showing in a study that when participants could alter some features of their face to look more attractive on digital images, they tended to make their eyes bigger [4]. This study however did not explore the link between self-face features and self-face perception of personality traits as done in face perception studies. In addition, this study involved static images and to our knowledge, similar work was not transposed to participants looking at moving mirror images of themselves. It therefore remains unclear how one perceives appeal, personality and intelligence of one's own face depending on specific facial features alterations, when looking at oneself on a mirrored video feedback.

The real-time modification of ones own self facial features could potentially distort senses of self identity beyond recognition. The ramifications of such illusions may be capable of inducing a type of self-uncanny valley, where users consider their mirrored faces to be merely the eerie resemblance of their own conceived self-identity. Indeed, previous works have highlighted the malleability of the self, showing that self-face representation can be altered to include another person's facial features [23]. It is therefore questionable how self-face feature alterations could affect self-identification, and how it could interfere with the perception of self facial traits like personality, appeal, intelligence and emotion. Augmented Reality offers the possibility to modify in real-time self-facial features and has already been the support of studies on self-perception.

\subsection{Augmented Reality and Self-Perception}

There have been a number of studies exploring self-perception through AR mirrors for marketing purposes (see [8] for a review). For instance, Javornik and Pizzetti [9] explored self-perception with an AR mirror having participants looking at themselves on a tablet with the possibility to superimpose virtual makeup. They showed that adding AR makeup could increase self-esteem and purchase intentions of users. Another study explored the impact of superimposed virtual sunglasses and found a relationship between participants body image perception and consumer experience (unfavourable body image would lead to better AR consumer experience) [34].

Other research investigated the use of AR mirrors in social media such as in SnapChat and Instagram that can also add virtual content on the face and sometimes distort its facial features. These studies are mainly part of the sociological field and explored for instance the long term impact of "beauty filters" on self-perception and self-esteem $[4,25]$. However, these studies either focused on investigating the effects of virtual face augmentation on consumers' self-concept and remained framed in the marketing context, or addressed the question of long-term effects of beauty filters from a sociological and psychological point of view. We believe that there is a lack of knowledge of the direct perception of the augmented self-face as seen through these types of AR mirrors. In particular, there is a gap in our knowledge around the roles played in perceiving personality traits, intelligence, appeal and emotion in our own distorted or modified faces, and the ways this differs from or resembles our perceptions of both real and virtual human faces.

\section{EXPERIMENT}

The main aim of this paper is to explore how users perceive appeal, personality, intelligence and emotion on their face when specific dis-

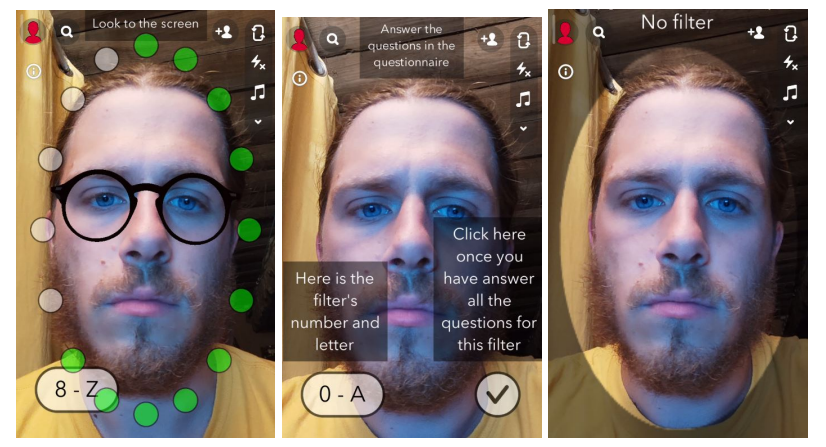

Figure 2: The experiment application interface. Left: Filter applied, with green circles indicating the time spent on the 7 seconds. Center: $\mathrm{UI}$ indicating to the participant that he must answer the questionnaire, and which letter they have to refer in it for this filter. Right: The 3 seconds no filter phase that was presented between each filter.

tortions are applied to it through AR filters. To do so, we conducted a remote experiment in which participants had to look at themselves through different filters and rate how much they agreed on several items related to self-face perception (see supplemental video for an example of a participant completing the experiment).

\subsection{Participants and Apparatus}

43 participants volunteered to take part in the experiment. After checking the data, 3 participants were removed because they reported incorrect filters order. All the remaining participants passed the data sanity check procedure detailed in Sect. 3.3.1. The remaining participants included 20 males and 20 females, from 20 to 50 years old $(M=29.02, S D=7.93)$. Most of them (32) described themselves as "White or Caucasian", 3 as "Asian or Pacific Islander", 1 as "Hispanic or Latino", 3 as "Multiracial or Biracial" and 1 as "Other".

Participants were recruited via general solicitations through internal emailing, mainly from the university campus. They were naive to the purpose of the experiment, and were not paid for their participation. They had normal or corrected-to-normal vision with lens (due to the distortions applied to the face, participants that could not see well without wearing glasses and that could not wear lenses could not participate). The study was conformed to the declaration of Helsinki. Before the experiment, participants were first briefed about the experiment, signed an informed-consent form and completed a demographic questionnaire.

Due to restrictions imposed by the Covid-19 pandemic, the experiment was conducted by participants remotely, without the help of an experimenter. The protocol was very detailed to ease the procedure. The experiment application was developed as a SnapChat lens (AR mask that overlay your face with details) with the Snap Inc. software Lens Studio. Participants had to download the SnapChat application on their smartphone or tablet, and create an account. To acquire the experiment lens, participants had access to a webpage in which they could scan an image through the SnapChat application which would directly lead them to the lens. A user interface on the lens (see Figure 2) would guide them through the procedure of the experiment, along with a form they had to complete in parallel on another device. The experiment was conducted in French and English, and participants performed the experiment in their native language.

\subsection{Filter Design}

Filters used in the experiment were designed considering literature findings on which facial features were likely to influence specific facial traits (see Section 2). Therefore, we decided to design filters that would modify respectively: the eye size, the eyes inter-distance 
and the width of the face and the orientation of the eyebrows. Each of these filters were changed in two directions (e.g., eye size could be either big or small) and two levels of intensity. In Figure 1 (A, B, $\mathrm{C}$ and $\mathrm{D}$ ), we show the variations of the eyes size modulation (from very small eyes to very big eyes). We introduced the two levels of intensity because we were interested in two questions: First, does the strength of the filter impact self-recognition? Second, does the strength of the filter, and potentially self-recognition, impact facial trait judgements of the self altered face? We also decided to include two filters that would not distort users' face but add virtual content on it, as it is a common feature in AR filters. One was aimed to be somewhat realistic and consisted in virtual glasses (see Figure 1, Q), and the other was aimed to be completely unrealistic consisting in a cat face effect with virtual cat ears and whiskers (see Figure 1, R).

The filters were designed in Lens Studio ${ }^{3}$, where it was possible to create specific facial alterations in different filters. The software allows to modify, in a proportional way, the distance between keypoints of the face ${ }^{4}$. This allows to adjust the filters to most faces morphology. The values of the deformations were defined via the state of the art data when it was possible, or empirically. In each case, the two intensity levels were chosen to be approximately twice each other and the low level could be called "subtle" while the high level was "obvious". We detail below the characteristics of the filters used:

In total, we designed 18 different filters:

- Eyes size: reduced/increased by $5 \%$ and $10 \%$ in horizontal, and $15 \%$ and $30 \%$ in vertical.

- Eyes inter-distance: reduced/increased by $10 \%$ and $20 \%$.

- FWHR: reduced/increased face width by $7.5 \%$ and $15 \%$.

- Eyebrows: very inward, inward, outward, very outward.

- Added elements: Virtual glasses and Cat face (ears and whiskers)

\subsection{Protocol}

Before the experiment, participants were given information to acquire the SnapChat lens necessary for the experiment and a link to a Google form. The Google form contained preliminary information for participants to get started on the experiment. Participants would also sign a consent form, complete a demographic questionnaire and they could then start the experiment. Participants were explained in the online form how to set the camera of their phone to front-facing mode, so they would see themselves, and they also received instructions about which distance should remain between their face and the camera so that it would be properly displayed in the application.

The procedure then consisted in having participants looking at themselves through different filters and answering self-perception ratings on the online form after each. For each filter, participants had to look at themselves onscreen for 7 seconds (some circles were displayed around the head of participants and would turn green one by one until the 7 seconds were done, see Figure 2, middle). Previous work has shown that people make trait judgements as quickly as $100 \mathrm{~ms}$ after seeing a face [21], but considering the originality of the setup of having people looking at themselves with face alterations, we decided to make the exposition longer up to 7 seconds. In addition, participants were asked to keep a neutral face when looking at themselves.

Each filter was identified with a UI on screen by its order in the experiment as well as a letter, and participants had to refer the corresponding letter in the form when ratings items about selfperception related to this filter (see Figure 2, right). The first filter participants would always start the experiment with, and the last filter they would end it with, were always "neutral" filters, i.e., their video feedback without any alteration. For both neutral filters participants would also rate the same items as for the altering filters,

${ }^{3}$ https://lensstudio.snapchat.com/

${ }^{4}$ https://lensstudio.snapchat.com/guides/face/face-effects/face-stretch/ which we aim to use as a baseline of participants self-perception, but also to evaluate if exposure to all our filters would impact their self-perception without altering filters. Furthermore, between each filter participants would see themselves without any alteration for 3 seconds, but also without needing to answer questions on the form after it. The aim was mainly to avoid the effect of transition from seeing oneself with one filter to another. In that spirit, we also randomized the order of filters for each participant, with a pseudo randomization that would randomize but control that there should not be two alterations in a row on the same facial feature (e.g., a filter making small eyes followed by a filter making big eyes). After answering ratings for all filters including the last neutral filter, participants had to answer a final short questionnaire that will be detailed in the next section. In total, the experiment lasted between 20 and 30 minutes.

\subsubsection{Collected Data}

In this experiment, we were interested in two main things. Firstly, we wished to determine if participants perceive personality, appeal, intelligence and emotion on their altered faces similarly to people tend to perceive those traits on other's faces. Secondly, to test if the intensity of the alterations would affect these results and self-recognition. In order to answer these questions, we asked participants to rate on a 7-points Likert scale how much they agreed with several statements for each filters (from Strongly Disagree to Strongly Agree) on an online form. The statements were all of the same form "I look like..." + a specific item. The items were divided in four categories (Personality, Appeal, Intelligence and Emotion) and were chosen in relation to previous work on face perception where facial features were found to be linked with specific traits.

In the Personality category, we included the items Trustworthy, Dominant and Aggressive. For Appeal, we used the items Attractive, Cute and Eerie. While the term "cute" is not as common as "attractive" in studies on face perception, cuteness is considered as a type of attractiveness [13]. We decided to include it as it is a term commonly associated with SnapChat filters, used to characterize for instance filters of dog or cat face and virtual glasses [14]. Eeriness has been more assessed in studies exploring the perception of virtual faces [5]. While looking at one's own face with AR alterations is different than looking at a fully virtual face, we believed that because in our experiment participants had to look at a modified version of their face, and not the face of someone else, they might perceive their faces with alteration as looking strange, because of the difference from their normal face. For this reason, we also asked participants to rate for each filter how much they agreed with the statement "this looks like me" on a similar 7-points Likert scale than other ratings. These two items were also important in order to assess the impact of the two levels of intensity of our filters. For Intelligence, we used the item Clever. For Emotion we decided to restrict on only two items, to avoid having too many variables. We used the items Angry and Sad as they were the most likely to be influenced by our eyebrows alterations. We also introduced two Control items: Beautiful and Ugly, with the aim to allow us to control if participants did not randomly answer the form. Indeed, the participants being autonomous and alone while doing the experiment, it was important to make sure it was done seriously without answering randomly. We used the results of these questions to remove participant who did not provide opposed answers for these items.

We also gathered demographic information (before starting the experiment) and personality (at the end of the experiment) data from participants. In terms of demographics, we were mainly interested in gender as previous work had highlighted differences between male and female participants in the way they perceived personality traits in faces [6]. As for personality, we were interested in participants' self-esteem as it was shown in previous work to interact with the perception of self-face alterations on images [4]. More precisely, 
Table 1: Main hypotheses of the study.

\begin{tabular}{|c|c|c|c|}
\hline Group & $\begin{array}{l}\text { Facial } \\
\text { Traits }\end{array}$ & Facial Features & Hypotheses \\
\hline Personality & Trustworthy & Face Width & P1: Wider male faces will be judged less trustworthy \\
\hline Personality & Trustworthy & Eyes Size & P2: Faces with bigger eyes perceived as more honest \\
\hline Personality & Trustworthy & Virtual Glasses & P3: Faces with virtual glasses will be judged as more trustworthy \\
\hline Personality & Dominant & Eye Size & P4: Faces with bigger eyes perceived less dominant \\
\hline Personality & Aggressive & Face Width & P5: Wider male faces will be rated more aggressive \\
\hline Personality & Aggressive & Eye Size & P6: Faces with smaller eyes perceived more aggressive \\
\hline Appeal & Attractive & Face Width & A1: Wider faces will be perceived less attractive \\
\hline Appeal & Attractive & Eyes Size & A2: Face with bigger eyes will be rated more attractive \\
\hline Appeal & Attractive & Virtual Glasses & A3: Face with virtual glasses will be rated less attractive \\
\hline Appeal & Cute & Eye Size & A4: Face with bigger eyes will be rated cuter \\
\hline Appeal & Cute & Cat Face & A5: Face with cat filter will be perceived cuter \\
\hline Appeal & Eeriness & All Filters & A6: Faces with low intensity alterations will not be rated as more eerie than normal face \\
\hline Appeal & Eeriness & All Filters & A7: Faces high intensity alterations will be rated as more eerie than normal face. \\
\hline Intelligence & Clever & Face Width & I1: Prolonged faces will be perceived as more intelligent \\
\hline Intelligence & Clever & Eyes inter-distance & I2: Wider distance between eyes will lead to higher perceived intelligence \\
\hline Intelligence & Clever & Virtual Glasses & I3: Faces with virtual glasses will be perceived as more clever \\
\hline Emotion & Angry & Eyebrows & E1: Faces with inward eyebrows will be perceived as angry \\
\hline Emotion & Sad & Eyebrows & E2: Faces with outward eyebrows will be perceived as sad \\
\hline Resemblance & $\begin{array}{l}\text { "This looks } \\
\text { like me" }\end{array}$ & All Filters & R1: Faces high intensity alterations will be rated as less looking like participants. \\
\hline Resemblance & $\begin{array}{l}\text { "This looks } \\
\text { like me" }\end{array}$ & All Filters & $\begin{array}{c}\text { R2: Faces with low intensity alterations will not be rated as less looking like } \\
\text { participants. }\end{array}$ \\
\hline
\end{tabular}

Felisberti and Musholt [4] found in their study that self-esteem was inversely correlated with eye, mouth and nose size manipulations made to increase one's attractiveness. We therefore used the same scale they used in their study (Rosenberg Self-Esteem Scale [27]) with the assumption that some differences in attractiveness ratings could be highlighted depending on participants self-esteem.

\subsection{Hypotheses}

We grouped our main hypotheses by our main trait categories: Personality, Appeal, Intelligence and Emotion (see Table 1). Our hypotheses regarding Personality were directly driven by previous work findings on face perception, with the assumption that participants would associate modulations of their face width and eyes size with judgements of trustworthiness, dominance and aggressiveness similarly as in previous face perception studies [6,10,11, 15, 22, 35] Our hypotheses regarding Appeal were similarly based on previous findings highlighting connections between attractiveness, cuteness and face width and eyes size. Yet, we also considered an additional hypothesis regarding our cat face filter, assuming that this type of filter, commonly characterize as "cute" in filters user community would elicit higher cuteness ratings. As for eerie, we did not expect specific facial features to impact this trait, but we expected that low intensity alterations would not be perceived as more eerie than normal face, while high intensity alterations would be. For Intelligence, we made the hypothesis that virtual glasses would make people rate themselves as more clever, based on Leder et al.'s study on the influence of glasses on perceived intelligence [15]. We also assumed based on previous work that narrower faces with wider eye interdistance would be perceived as more clever [12]. For Emotion, we hypothesised that faces with inward eyebrows would be perceived as angry and faces with outward eyebrows would be perceived as sad. Furthermore, we hypothesised that participants would still rate that their face looked like them with low intensity alterations, and that they would rate that their face no longer looked like them with high intensity alterations, regardless of filters. Finally, we expected gender to impact the self-face perception as it did in previous work (e.g. only wider male faces were rated more aggressive [6]). We also expected self-esteem scores to interfere with attractiveness ratings along with eye size modulation [4].

\section{Results}

\subsection{Statistical analysis}

We computed the answer of the participants as a numeric independent variable ranging from 1 to 7 . The construction of the experiment (by evaluating a reference filter) and a statistical analysis taking into account the within-subject pattern of the experiment allows to measure the difference in perception between the "normal" face and the distorted version, which limits the noise in the data interpretation. Due to the robustness of these method regarding not normally distributed data, when applied to Likert-scale analysis, and to prevent a loss of information $[18,20]$, we used parametric methods to analyse our results. First, we computed a one-way Analysis of Variance (ANOVA) for each item, considering the filter as a within-subjects categorical variable. Upon significance, we conducted per-group analysis using Tukey's HSD tests. To evaluate the effect of gender (resp. self-esteem), we conducted two-way ANOVA, using gender as a factorial factor (resp. the self-esteem score as a continuous variable).

To help distinguish between effects due to links between items and filter effects, a correlation matrix was also computed between the score for each item, using the Pearson method.

\subsection{Filters effect}

When analyzing the results, we found a main effect of the filters for all the one-way ANOVAs conducted for each item (with $p<0.001$ ). The per-group analysis of the Tukey HSD tests highlighted numerous 


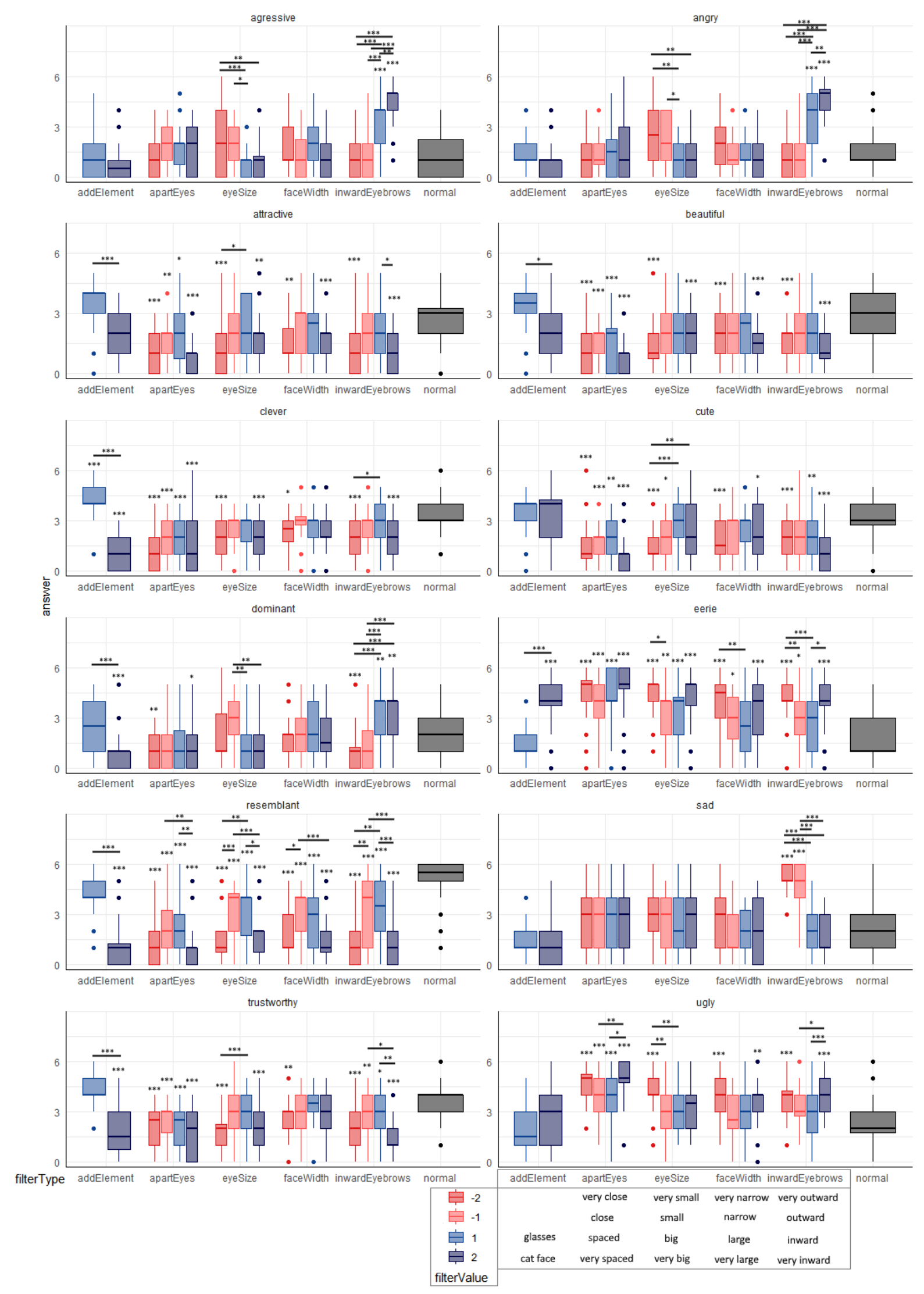

Figure 3: Participants answers, for each item, grouped by filter type. Significance of results from the Tuckey HSD post hoc analysis are reported as stars. Comparison between the normal face and each filter is reported above the filter's results, and other comparison are reported with brackets. Only significant results among two filters of the same group are reported. 


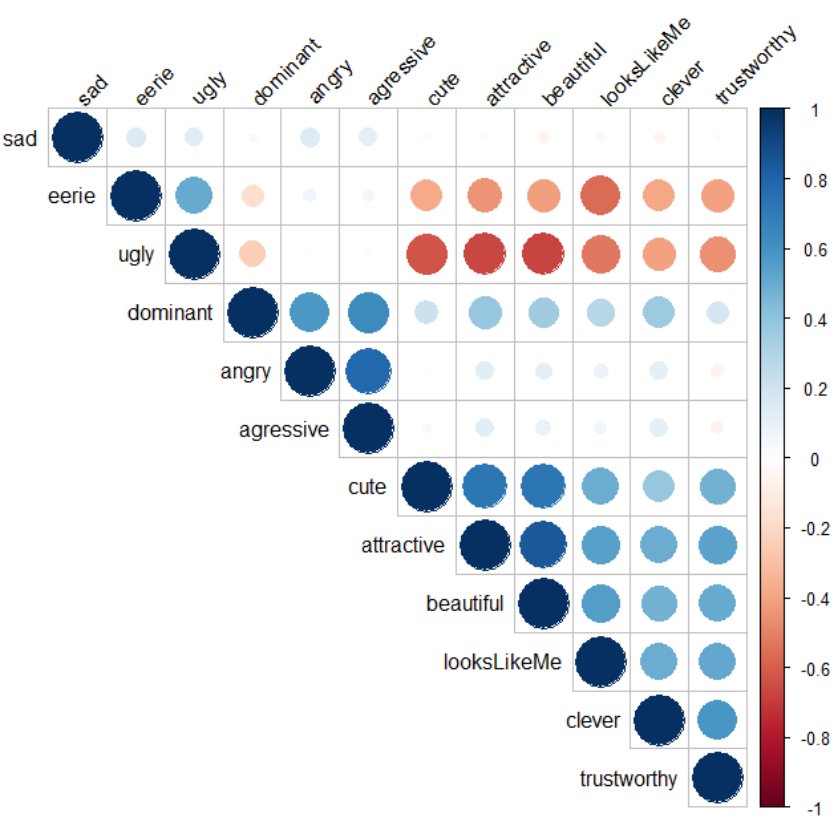

Figure 4: Correlation matrix between the score for each item, using the Pearson's correlation coefficient.

significant effects reported in Fig. 3. We choose to report only the significant differences between two filters impacting the same feature (e.g. between small eyes and big eyes) or between one feature and the normal face. Comparisons between filters impacting different features were then not reported.

\subsection{Gender effect}

As for the one-way ANOVAs, the main effect of the filter was found significant across all items.

The two-way ANOVA reported a main effect of gender for "attractive" $(p=0.036)$, "eerie" $(p=0.044)$, "beautiful" $(p=0.044)$ and "resemblance" $(p=0.009)$. Male participants tended to rate themselves as more attractive, less eerie and more beautiful than female. We also reported interaction effects between gender and the filters for "eerie" $(p=0.044)$ and "resemblance" $(p=0.026)$. Pairwise comparisons for "eerie", conducted with the Holm-Bonferroni correction, showed no significant effect. Nevertheless, they showed interesting trends where females were more impacted by eye position changes $(p=0.050$ for sightly closer eyes and $p=0.092$ for very distant eyes). The pairwise comparisons for the "resemblance" item showed that females felt less resemblance to themselves with smaller eyes $(p=0.019)$.

\subsection{Self-esteem effect}

As before, the main effect of the filters was found significant across all items. However, no other effect (main or interaction effect) was found including the self-esteem score of the participant.

\subsection{Correlation between items}

The correlation matrix between items is presented in Fig. 4. Results show a positive correlation $(>0.3)$ between "ugly" and "eerie" as well as a positive correlation between "dominant", "angry" and "aggressive". Finally, a positive correlation was found between "resemblance", "cute", "attractive", "beautiful", "clever", "trustworthy" and a negative correlation between these items and "ugly" and "eerie".

\section{Discussion}

\subsection{Personality}

Our results show an impact of face width on participants self-reports of trustworthiness, with very narrow faces being less trustworthy, which therefore partly validates P1. However, it is worth noting that the eye enlargement filters have a trustworthiness comparable to the normal face, unlike all the other filters. Though, $\mathbf{P 2}$ is not validated as self-faces with bigger eyes were not perceived as more honest. Another interesting effect was found: if the size of eyes is altered, independently of making them bigger or smaller, users will rate their face as less trustworthy. This pattern was also identified for the alteration of inter-distance between the eyes, and eyebrows. One could therefore assume that the positive effect of eye size would be counterbalanced by a general negative effect of the filters. Virtual Glasses did not influence trustworthiness ratings, rejecting P3. For dominance, we found that bigger eyes were perceived less dominant than smaller eyes which validates P4. However, bigger eyes were not found different than normal face. We also found that inward eyebrows were perceived as more dominant, which was not part of our main hypotheses but was expected. Similarly, we found that the cat-face filter made participants perceive themselves less dominant than without. As for aggressive, our results suggest that participants judged their face as more aggressive with smaller eyes, which validates P6. Interestingly, results were only true when comparing one's face with smaller eyes and one's face with bigger eyes, but not comparing one's normal face with one's altered face (with smaller or bigger eyes). This may suggest that the cognitive process of comparison between two modified self-faces is different than between ones normal self-face and ones modified self-face. Furthermore, face width did not impact self-face perception of aggressiveness, rejecting $\mathbf{P 5}$.

\subsection{Appeal}

Our results on attractiveness did not show a strong impact of any of the facial alterations, eye size and face width included, rejecting therefore hypotheses A1 and A2. What was noted however is that independently of the direction of the alterations, changes to eyes size, eye inter-distance, eyebrows and face width tended to make participants perceive themselves as less attractive. This result is quite unexpected, and more precisely we were surprised that participants did not find themselves more attractive with bigger eyes, since this hypothesis was highly encouraged by the study of Felisberti et al where participants tended to make their eyes bigger on their face to look more attractive [4], and by the common characterisation of "beauty filters" that make the eyes bigger $[4,25]$. While we tried to design our eyes size alteration similarly to the common "beauty filters", it would be interesting to explore in future work different type of eye size alterations and their effect on self-attractiveness reports. Furthermore, Virtual Glasses did not influence attractive ratings, rejecting A3. As for cute, we did not find the expected effect of the cat-face filter on cute ratings. However, unlike attractive ratings, eyes size impacted ratings of cuteness. Faces with smaller eyes were perceived as less cute. However, results do not support that faces with bigger eyes were symmetrically perceived cuter, which therefore does not allow us to fully validate A5. Regarding eerie, nearly all our face alterations were perceived as more eerie than participants normal face, which was somehow unexpected, as we thought less intense alterations would reduce the eeriness impact. This is nonetheless true for only one intensity of face width: a face a bit wider was not rated more eerie, while a face a bit narrower was already rated more eerie compared to the normal face. Moreover, we showed trends that females were more impacted by eye position changes, suggesting that some modification of the face would be perceived as more or less acceptable according to gender. Virtual glasses also did not impact the results on eeriness, while cat face did. Interestingly, this result suggests that realistic AR- 
added-content does not seem to disrupt the appeal of one's own face, while unrealistic AR-added-content seem to increase eeriness perception towards one's own face. Yet, we acknowledge that we only considered two different examples of this type of added content AR filters, and more of them should be considered to really address their potential influence on eeriness ratings.

Overall, the decrease in appeal perception of faces using filters, and the fact that strong correlations were found between "resemblance" and many appeal traits suggests that users are disturbed by the differences between their modified face and their normal face, which reduces their perceived appeal.

\subsection{Intelligence}

Virtual glasses strongly influenced intelligence perception in participants self-face, as participants rated themselves as more clever with virtual glasses than without, validating I3. While other assumptions related to the virtual glasses were not verified, it is interesting that virtual glasses have the same effect as real glasses on perceived intelligence when added on the face with AR filters. Face width was shown to have an effect on perceived intelligence, as participants rated their own face as less clever when more narrow, which is the contrary to previous work. Nevertheless, eyes inter-distance was not found to influence as expected the perceived intelligence, as both closer and wider eyes were shown to decrease the perceived intelligence.

\subsection{Emotion}

As expected, eyebrow orientation had a strong impact on emotion recognition, faces with inward eyebrows being perceived as more angry than normal faces, and faces with outward eyebrows being perceived as more sad, supporting E1 and E2.

\subsection{Resemblance}

Results highlighted that participants were always good at noticing the difference between their own face and their face with filters. Indeed, even when the alteration was of low intensity, participants rated that they looked significantly different with filters than without, which therefore rejects (R1). However, this is true for all filters except one: the virtual glasses. It is even more interesting that virtual glasses did not impact participants self-recognition, while cat face filter, which is also a filter that does not distort but only adds content on top of the face, did impact self-recognition. We believe future work would be interesting to conduct in order to investigate the impact of AR add-on filters realism on self-recognition.

\subsection{Influence of gender}

Results highlighted that male participants tended to rate themselves as more attractive, less eerie and more beautiful than female participants did. In addition, male participants tended to rate that they looked more like themselves with filters applied than female participants did. An interesting trend was also highlighted showing that female participants were more impacted by eye position changes than male. An impact was also found of gender on the way participants found that they looked like themselves with filters or not: female participants were more unlikely to feel that they looked like themselves with smaller eyes than male participants. It is difficult to understand why gender seemed to impact several self-perception dimensions in this context. Previous research on face perception highlighted that an effect of eye size alteration was only significant for female participants [7], which is in line with our results and may suggest that women are more sensitive to change in their eyes. The fact that women are more used to Snapchat filters than men (in 2021, the percentage of women using SnapChat worldwide is of $57,4 \%$ and for $40,9 \%$ of men $^{5}$ ) may have impacted the way they

\footnotetext{
${ }^{5}$ https://www.statista.com/statistics/326460/snapchat-global-gendergroup/
}

perceive themselves through them. Overall, our results suggest that some modification to the face would be perceived as more or less acceptable according to gender. This could partly explain the impact found of gender on certain traits.

\subsection{Limitations and Future Work}

First, our participants were mainly self reported as "White or Caucasian". As such, more studies are needed to assess the effect of AR filters for other ethnicities.

Then, results showed that participants usually found in themselves less resemblance when using AR filters. To mitigate this effect, it would be interesting in future work to use feedback to improve the sense of ownership of modified faces. Visuomotor feedback could be used by making participants perform expressions with their face, and visuotactile by making them touch their face. We believe both effects could contribute to a stronger enfacement illusion towards one self altered face [30]. It would also be interesting to measure this sense of ownership in order to measure a possible correlation with the effect of filters in AR. Also, we focused here on the perception only of one's own modified face, but one could also compare these measurements to other measurements made by displaying faces of other people. This would also allow to verify if the face alterations made with our filters elicit similar perception of others' faces as in previous work. In the present study, we were focused on how participants rated how they looked like. In future work, we envision investigating how participants feel when they use these filter. In Virtual Reality, research highlighted how self-representation alterations could lead to self-concept and behaviour modification [33]. Using the findings of this experiment, it is then possible to propose filters modifying their self-perception in order to further explore if AR filters can influence human behaviour.

\section{Conclusion}

In this paper, we explored for the first time the self-face perception of personality, intelligence, emotion and appeal traits with the use of AR filters. Our results highlight that there is a similarity in the way we perceive some facial traits in others and in our own face. In addition, even small distortions of the face impacted participants selfrecognition, reinvigorating the idea that humans are highly attentive to faces and to their own image in particular. We reported various effects consistent with the state of the art, showing that features influencing judgment can be applied to self-perception. In particular, we observed that eye size was the most important parameter, affecting the self-perceived aggressiveness, anger, cuteness and dominance of the users. Eyebrows orientation was also very effective to convey emotions as sadness and anger. However, our results also revealed specific effects of AR filters, which show that these effects can be reduced in this particular situation, and even more so when the user no longer recognizes himself in the image. Especially, the more the face is distorted, the less resembling and the more eerie the image of the person is considered, which has a negative impact on the perception of all the usually positive traits.

It is commonly said that today's communication is happening more and more through screens, increasing greatly our exposure to a video feedback of ourselves. This is even more true in these recent times of pandemic. In this context, and considering the important use of filters in these communication means, it seems important to evaluate the impact of such a distorted representation of ourselves, and we believe that the results of the present study lay the first foundations for research to evaluate the psychological impact of AR filters on self-perception.

\section{ACKNOWLEDGMENTS}

This work was conducted with the financial support of the RADICal project (Grant No. 19/FFP/6409). 


\section{REFERENCES}

[1] J. M. Carré, C. M. McCormick, and C. J. Mondloch. Facial structure is a reliable cue of aggressive behavior. Psychological Science, 20(10):1194-1198, 2009. PMID: 19686297. doi: 10.1111/j.1467-9280 .2009.02423.x

[2] J. A. Diego-Mas, F. Fuentes-Hurtado, V. Naranjo, and M. Alcañiz. The influence of each facial feature on how we perceive and interpret human faces. i-Perception, 11(5):2041669520961123, 2020. PMID: 33062242. doi: 10.1177/2041669520961123

[3] K. Edwards. Effects of sex and glasses on attitudes toward intelligence and attractiveness. Psychological Reports, 60(2):590-590, 1987. doi 10.1177/003329418706000201

[4] F. M. Felisberti and K. Musholt. Self-face perception: Individual differences and discrepancies associated with mental self-face representation, attractiveness and self-esteem. Psychology \& Neuroscience, 7(2):65-72, 2014. doi: 10.3922/j.psns.2014.013

[5] Y. Ferstl and R. McDonnell. A perceptual study on the manipulation of facial features for trait portrayal in virtual agents. In Proceedings of the 18th International Conference on Intelligent Virtual Agents, IVA '18, p. 281-288. Association for Computing Machinery, New York, NY, USA, 2018. doi: 10.1145/3267851.3267891

[6] S. N. Geniole, T. F. Denson, B. J. Dixson, J. M. Carré, and C. M. McCormick. Evidence from meta-analyses of the facial width-toheight ratio as an evolved cue of threat. PLOS ONE, 10(7):1-18, 07 2015. doi: 10.1371/journal.pone. 0132726

[7] G. Gonçalves, A. Gomes, M. Ferrão, T. Parreira, J. Santos, J.-C. Giger and A. Martins. Once upon a face: the effect of eye size, observer and stimulus gender on impression formation. Current Psychology, 07 2014. doi: 10.1007/s12144-014-9244-3

[8] A. Javornik, B. Marder, M. Pizzetti, and L. Warlop. Augmented self - the effects of virtual face augmentation on consumers' self-concept. Journal of Business Research, 130:170-187, 2021. doi: 10.1016/j. jbusres.2021.03.026

[9] A. Javornik and M. Pizzetti. "mirror mirror on the wall, who is real of them all?" -the role of augmented self, expertise and personalisation in the experience with augmented reality mirror. 012017.

[10] C. F. Keating. Gender and the physiognomy of dominance and attractiveness. Social Psychology Quarterly, 48(1):61-70, 1985.

[11] H. M. Kleider-Offutt, A. D. Bond, S. E. Williams, and C. J. Bohil. When a face type is perceived as threatening: Using general recognition theory to understand biased categorization of afrocentric faces. Memory \& Cognition, 46(5):716-728, Jul 2018. doi: 10.3758/s13421-018-0801 $-0$

[12] K. Kleisner, V. Chvátalová, and J. Flegr. Perceived intelligence is associated with measured intelligence in men but not women. PLOS ONE, 9(3):1-7, 03 2014. doi: 10.1371/journal.pone.0081237

[13] K. Kuraguchi and K. Kanari. Face inversion effect on perceived cuteness and pupillary response. Frontiers in Psychology, 11:2316, 2020 doi: $10.3389 /$ fpsyg. 2020.558478

[14] C. Lavrence and C. Cambre. "do i look like my selfie?": Filters and the digital-forensic gaze. Social Media + Society, 6(4):2056305120955182, 2020. doi: $10.1177 / 2056305120955182$

[15] H. Leder, M. Forster, and G. Gerger. The glasses stereotype revisited: Effects of eyeglasses on perception, recognition, and impression of faces. Swiss Journal of Psychology / Schweizerische Zeitschrift für Psychologie / Revue Suisse de Psychologie, 70(4):211-222, 2011. doi 10.1024/1421-0185/a000059

[16] A. C. Little, D. M. Burt, and D. I. Perrett. What is good is beautiful: Face preference reflects desired personality. Personality and Individual Differences, 41(6):1107-1118, 2006. doi: 10.1016/j.paid.2006.04.015

[17] A. C. Little and D. I. Perrett. Using composite images to assess accuracy in personality attribution to faces. British Journal of Psychology, 98(1):111-126, 2007. doi: 10.1348/000712606X109648

[18] C. Mircioiu and J. Atkinson. A Comparison of Parametric and NonParametric Methods Applied to a Likert Scale. Pharmacy, 5(4):26, may 2017. doi: 10.3390/pharmacy5020026

[19] M. Mori, K. MacDorman, and N. Kageki. The uncanny valley [from the field]. IEEE Robotics and Automation Magazine, 19:98-100, 06 2012. doi: 10.1109/MRA.2012.2192811
[20] G. Norman. Likert scales, levels of measurement and the "laws" of statistics. Advances in Health Sciences Education, 15(5), 2010. doi: 10 .1007/s10459-010-9222-y

[21] N. N. Oosterhof and A. Todorov. Shared perceptual basis of emotional expressions and trustworthiness impressions from faces. Emotion, 9(1):128-133, 2009. doi: 10.1037/a0014520

[22] M. E. Ormiston, E. M. Wong, and M. P. Haselhuhn. Facial-width-toheight ratio predicts perceptions of integrity in males. Personality and Individual Differences, 105:40-42, 2017. doi: 10.1016/j.paid.2016.09. 017

[23] G. Porciello, I. Bufalari, I. Minio-Paluello, E. Di Pace, and S. M. Aglioti. The 'enfacement' illusion: A window on the plasticity of the self. Cortex, 104:261-275, 2018. Special Section: The body and cognition: the relation between body representations and higher level cognitive and social processes. doi: 10.1016/j.cortex.2018.01.007

[24] M. Przylipiak, J. Przylipiak, R. Terlikowski, E. Lubowicka, L. Chrostek, and A. Przylipiak. Impact of face proportions on face attractiveness. Journal of Cosmetic Dermatology, 17(6):954-959, 2018. doi: 10.1111/ jocd. 12783

[25] S. Rajanala, M. B. C. Maymone, and N. A. Vashi. Selfies-living in the era of filtered photographs. JAMA Facial Plastic Surgery, 20(6):443444, 2018. PMID: 30073294. doi: 10.1001/jamafacial.2018.0486

[26] B. Rooney, H. Keyes, and N. Brady. Shared or separate mechanisms for self-face and other-face processing? evidence from adaptation. Frontiers in Psychology, 3:66, 2012. doi: 10.3389/fpsyg.2012.00066

[27] M. Rosenberg. Rosenberg self-esteem scale (rse). Acceptance and commitment therapy. Measures package, 61(52):18, 1965.

[28] N. Sagiv and S. Bentin. Structural Encoding of Human and Schematic Faces: Holistic and Part-Based Processes. Journal of Cognitive Neuroscience, 13(7):937-951, 10 2001. doi: 10.1162/089892901753165854

[29] M. Stirrat and D. Perrett. Valid facial cues to cooperation and trust: Male facial width and trustworthiness. Psychological Science, 21(3):349-354, 2010. PMID: 20424067. doi: 10.1177/ 0956797610362647

[30] A. Tajadura-Jiménez, M. R. Longo, R. Coleman, and M. Tsakiris. The person in the mirror: using the enfacement illusion to investigate the experiential structure of self-identification. Consciousness and cognition, 21(4):1725-1738, Dec 2012. 23123685[pmid]. doi: 10. 1016/j.concog.2012.10.004

[31] Y. Wang, J. Geigel, and A. Herbert. Reading personality: Avatar vs. human faces. In 2013 Humaine Association Conference on Affective Computing and Intelligent Interaction, pp. 479-484, 2013. doi: 10. 1109/ACII.2013.85

[32] M. Wegrzyn, M. Vogt, B. Kireclioglu, J. Schneider, and J. Kissler. Mapping the emotional face. how individual face parts contribute to successful emotion recognition. PLOS ONE, 12(5):1-15, 05 2017. doi: 10.1371/journal.pone.0177239

[33] N. Yee and J. Bailenson. The proteus effect: The effect of transformed self-representation on behavior. Human Communication Research, 33:271 - 290, 07 2007. doi: 10.1111/j.1468-2958.2007.00299.x

[34] M. Y.-C. Yim and S.-Y. Park. "i am not satisfied with my body, so i like augmented reality (ar)": Consumer responses to ar-based product presentations. Journal of Business Research, 100:581-589, 2019. doi: 10.1016/j.jbusres.2018.10.041

[35] L. A. Zebrowitz, L. Voinescu, and M. A. Collins. "wide-eyed" and "crooked-faced": Determinants of perceived and real honesty across the life span. Personality and Social Psychology Bulletin, 22(12):12581269, 1996. doi: 10.1177/01461672962212006 